EESTI NSV TEADUSTE AKADEEMIA TOIMETISED. XI KOIDE

FOOSIKALIS-MATEMAATILISTE JA TEHNILISTE TEADUSTE SEERIA. 1962, NR. I

ИЗВЕСТИЯ АКАДЕМИИ НАУК ЭСТОНСКОН ССР. ТОМ ХІ СЕРИЯ ФИЗИКО-МАТЕМАТИЧЕСКИХ И ТЕХНИЧЕСКИХ НАУК. 1962, № 1

\title{
О ФОКУСИРУЮЩИХ СВОЙСТВАХ СЕКТОРНОГО ЦИКЛОТРОНА
}

\author{
Ю. ЛЕМБРА
}

\section{1. Введение}

В настоящей работе предложенный Балдиным, Михайловым и Рабнновнчем метод огибающих $\left[{ }^{1,2}{ }^{2}\right]$ применяется для исследования фокусирующих свойств секторного циклотрона $\left[{ }^{3},{ }^{4}\right]$. Целесообразность применения метода огибающих в данном случае вытекает из того обстоятельства, что с его помощью можно легко сравнить фокусировку в различных типах ускорителей.

Нас не будет интересовать какой-либо конкретный секторный циклотрон. Поэтому объектом исследования выбран простейший (по принципу) вариант секторного циклотрона, элемент периодичности магнитной системы которого состоит из двух секторов с различными значениями напряженности однородного магнитного поля: $H$ (в дальнейшем сектор 1) и $x H(x<1$, сектор 2). Такой подход удобен для выяснения характерных особенностей фокусирующих свойств секторного циклотрона, не затемненных посторонними эффектами.

В ['] и [2] метод огибающих развит для случая непрерывности радиальной и вертикальной скоростей частицы при переходе от одной составной части элемента периодичности к другой и от одного элемента периодичности к другому. В данном случае радиальная и вертикальная скорости частицы изменяются скачкообразно при переходе из одного сектора в другой. Однако, как будет показано ниже, путем рассмотрения переходной области малой длины $l$ можно и в предельном случае $l \rightarrow 0$ (чему соответствует скачкообразное изменение скорости) применить соответствующие фор-

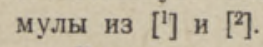

Выпишем из ['] и [ㄹ] основные формулы для применения метода огибающих. Бетатронные колебания описываются уравнениями типа

$$
\frac{d^{2} y}{d s^{2}}+g(s) y=0, \quad g(s+L)=g(s) .
$$

В уравнении (1) означает $y$ радиальное (@) или вертикальное (z) отклонение от равновесной орбиты; $s$ - длнна дуги равновесной орбиты; $L$ - длина элемента периодичности магнитной системы, измеренная по равновесной орбите. Огибающей траектории частиц является кривая

где

$$
y(s)= \pm F(s)
$$

$$
F^{2}(s)=\frac{\Phi(s)}{\Phi_{0}}\left\{y_{0}^{2}+\Phi_{0}^{2}\left\lceil\left(\frac{d y}{d s}\right)_{0}-\frac{y_{0}}{2}\left(\frac{d \ln \Phi}{d s}\right)_{0}\right]^{2}\right\}
$$

Здесь индекс 0 введен для обозначения начальных значений при $s=s_{0}$; $(s)-$ квадрат модуля функции Флоке, нормированной так, чтобы вронскнан нормальных рєшений $\Psi(s)$ и $\Psi^{*}(s)$ 


$$
W=\left|\begin{array}{cc}
\Psi & \psi * \\
\frac{d \Psi}{d s} & \frac{d \Psi *}{d s}
\end{array}\right|=\frac{2}{i}
$$

Қак известно (см., напр., [5]), нормальные решения обладают свойством

$$
\Psi(s+L)=e^{i \mu} \Psi(s)
$$

( $\mu$ - характеристический показатель) и имеют такой же модуль, как и функция Флоке.

Максимальное значение $\Phi(s)$ принято называть эффективным радиусом ускорителя $[1,2]$

$$
R_{э \phi \phi}=\max \Phi(s)
$$

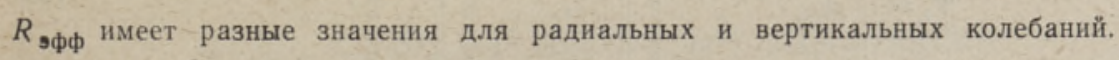

Имея в виду важную роль, которую играет функция $\Phi(s)$ в методе огибающих. наше внимание в дальнейшем обращено на изучение ее поведения.

\section{2. Радиальные колебания}

В первом приближении можно считать для уравнения (1)

$$
g_{r}(s)=\left\{\begin{array}{cl}
\frac{1}{r^{2}} & \text { в секторе 1 } \\
-\frac{(1-x) \operatorname{tg} \gamma_{21}}{r l_{21}} & \text { в переходной области из сектора 1 } \\
\frac{x^{2}}{r^{2}} & \text { в секторе } 2 \\
-\frac{(1-x) \operatorname{tg} \gamma_{12}}{r l_{12}} & \text { в переходной области из сектора 2 } 2
\end{array}\right\}
$$

В уравнении (7) $r$ - радиус равновесной орбиты в секторе $1 ; l_{12}$ и $l_{21}$ - длины переходных областей, измеренных вдоль равновесной орбиты, которую мы считаем в переходной области прямой при условии $l_{12}, l_{21} \ll r\left(v_{1}+\frac{v_{2}}{\varkappa}\right)=L \quad\left(v_{1}\right.$ и $v_{2}-$ углы поворота равновесной орбиты в секторах 1 и 2); $\gamma_{12}$ и $\gamma_{21}$ - углы между касательной к равновесной орбите и нормалью к граничной линин, отделяющей сектор опереходной области. Знак угла $\gamma$ определяем по принятому в $\left.{ }^{3}\right]$ правилу.

Составление точных дифференциальных уравнений движения в переходной области возможно на основе данных магнитных измерений. Однако с учетом результатов работы [6] мы можем при выводе (7) аппроксимнровать ход краевого магнитного поля линейной функцией.

Нормальное решение (1) найдем сшиванием. Вычисления удобно провести в матричной форме. При этом приняты следующие сокращения:

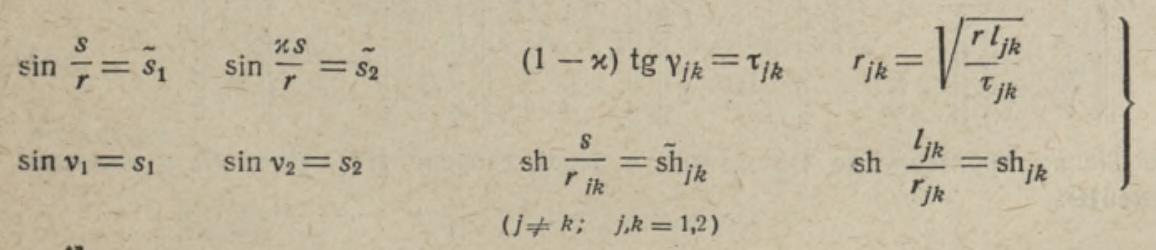


Аналогичные обозначения введены также для косинусов. В зависимости от знака $\gamma_{j k}$ величина $r_{j k}$ может быть вещественной или мнимой. Отсчет длины $s$ проводится п формулах (8) от начала сектора и от начала переходной области.

При этих обозначениях решение (1) принимает вид:

в секторе 1

$$
\vec{\Psi}_{r}=\left\|\begin{array}{c}
\Psi_{r} \\
\frac{d \Psi_{r}}{d s}
\end{array}\right\|=\left\|\begin{array}{cc}
\tilde{c}_{1} & r \tilde{s}_{1} \\
-\frac{\tilde{s}_{1}}{r} & \tilde{c}_{1}
\end{array}\right\|\left\|\begin{array}{c}
C_{r} \\
D_{r}
\end{array}\right\|
$$

в переходной области из сектора 1 в сектор 2

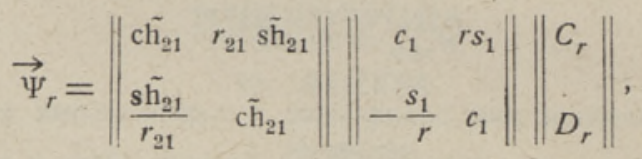

в секторе 2

$$
\vec{\Psi}_{r}=\left\|\begin{array}{cc}
\tilde{c}_{2} & \frac{r}{\varkappa} \tilde{s}_{2} \\
-\frac{\varkappa}{r} \tilde{s_{2}} & \tilde{c_{2}}
\end{array}\right\|\left\|\begin{array}{ll}
\mathrm{ch}_{21} & r_{21} \mathrm{sh}_{21} \\
\frac{\mathrm{sh}_{21}}{r_{21}} & \mathrm{ch}_{21}
\end{array}\right\|\left\|\begin{array}{cc}
c_{1} & r s_{1} \\
-\frac{s_{1}}{r} & c_{1}
\end{array}\right\|\left\|\begin{array}{c}
C_{r} \\
D_{r}
\end{array}\right\|
$$

и в переходной области из сектора 2 в сектор 1

$$
\vec{\Psi}_{,}=\left\|\begin{array}{cc}
c \tilde{h}_{12} & r_{12} \mathrm{sh}_{12} \\
\frac{\tilde{h}_{12}}{r_{12}} & c \tilde{h}_{12}
\end{array}\right\|\left\|\begin{array}{cc}
c_{2} & \frac{r s_{2}}{\varkappa} \\
-\frac{\gamma s_{2}}{r} & c_{2}
\end{array}\right\|\left\|\begin{array}{cc}
c h_{21} & r_{21} s_{21} \\
\frac{s h_{21}}{r_{21}} & c h_{21}
\end{array}\right\|\left\|\begin{array}{cc}
c_{1} & r s_{1} \\
-\frac{s_{1}}{r} & c_{1}
\end{array}\right\|\left\|\begin{array}{c}
C_{r} \\
D_{r}
\end{array}\right\|,
$$

где $C_{r}$ и $D_{r}-$ постоянные, которые определяются из условий (4) и (5).

Переходим к рассмотрению случая, когда радиальная скорость изменяется скач. кообразно при переходе из одного сектора в другой. Для этого следует полагать в предыдущих формулах $l_{12} \rightarrow 0$ и $l_{21} \rightarrow 0$. Тогда матрицы, описывающие движение в переходной области, принимают форму:

$$
\left\|\begin{array}{cc}
1 & 0 \\
\frac{\tau_{12}}{r} & 1
\end{array}\right\| ; \quad\left\|\begin{array}{cc}
1 & 0 \\
\frac{\tau_{21}}{r} & 1
\end{array}\right\|
$$

Согласно этому решение в секторе 2 принимает вид:

$$
\vec{\Psi}_{r}=\left\|\begin{array}{cc}
\tilde{c}_{2} & \frac{r \tilde{s}_{2}}{x} \\
-\frac{\chi \tilde{s}_{2}}{r} & \tilde{c}_{2}
\end{array}\right\|\left\|\begin{array}{cc}
1 & 0 \\
\frac{\tau_{21}}{r} & 1
\end{array}\right\|\left\|\begin{array}{cc}
c_{1} & r s_{1} \\
-\frac{s_{1}}{r} & c_{1}
\end{array}\right\|\left\|\begin{array}{c}
C_{r} \\
D_{r}
\end{array}\right\|
$$

а из (5) и (12) вытека̀ет

$$
\left\|\begin{array}{cc}
1 & 0 \\
\frac{\tau_{21}}{r} & 1
\end{array}\right\|\left\|\begin{array}{cc}
c_{2} & \frac{r s_{2}}{x}
\end{array}\right\|\left\|\begin{array}{cc}
1 & 0 \\
-\frac{\gamma s_{2}}{r} & c_{2}
\end{array}\right\|\left\|\begin{array}{cc}
c_{1} & r s_{1} \\
\frac{\tau_{21}}{r} & 1
\end{array}\right\|\left\|\begin{array}{cc}
C_{r} \\
-\frac{s_{1}}{r} & c_{1}
\end{array}\right\|\left\|\begin{array}{c}
D_{r}
\end{array}\right\|=e^{i \mu_{r}}\left\|\begin{array}{c}
C_{r} \\
D_{r}
\end{array}\right\| .
$$

Обозначим пронзведение 4-х матриц в левой стороне (14) через $A_{r}$ и выпишем ее элементы: 


$$
\begin{aligned}
& \left(A_{r}\right)_{11}=c_{1} c_{2}+\frac{\tau_{21} c_{1} s_{2}}{x}-\frac{s_{1} s_{2}}{x} \\
& \frac{\left(A_{r}\right)_{12}}{r}=s_{1} c_{2}+\frac{\tau_{21} s_{1} s_{2}}{x}+\frac{c_{1} s_{2}}{x} \\
& r\left(A_{r}\right)_{21}=-s_{1} c_{2}-x c_{1} s_{2}+\tau_{12}\left(c_{1} c_{2}-\frac{s_{1} s_{2}}{x}\right)+\tau_{21} c_{1} c_{2}+\frac{\tau_{12} \tau_{21}}{\varkappa} c_{1} s_{2} \\
& \left(A_{r}\right)_{22}=c_{1} c_{2}-x s_{1} s_{2}+\tau_{12}\left(s_{1} c_{2}+\frac{c_{1} s_{2}}{\varkappa}\right)+\tau_{21} s_{1} c_{2}+\frac{\tau_{12} \tau_{21}}{\varkappa} s_{1} s_{2} .
\end{aligned}
$$

Из (14) и (15) найдем:

$$
\begin{gathered}
\cos \mu_{r}=\frac{\left(A_{r}\right)_{11}+\left(A_{r}\right)_{22}}{2}= \\
=c_{i} c_{2}-\frac{1+x^{2}}{2 x} s_{1} s_{2}+\frac{\tau_{12}+\tau_{21}}{2}\left(s_{1} c_{2}+\frac{c_{1} s_{2}}{x}\right)+\frac{\tau_{12} \tau_{21} s_{1} s_{2}}{2 x} \\
D_{r}=\frac{\beta_{r}}{r} C_{r} \\
\beta_{r}=\frac{r\left(e^{i \mu_{r}}-\left(A_{r}\right)_{11}\right)}{\left(A_{r}\right)_{12}} .
\end{gathered}
$$

Постоянную $C_{r}$ определяем из (4)

$$
C_{r}=\sqrt{\frac{\left(A_{r}\right)_{12}}{\sin \mu_{r}}}
$$

Таким образом, мы получим нормальное решение в следующей форме:

B секторе 1

$$
\Psi_{r}(s)=\sqrt{\frac{\left(A_{r}\right)_{12}}{\sin \mu_{r}}}\left(\tilde{c}_{1}+\beta_{r} \tilde{s}_{1}\right) \text {. }
$$

в секторе 2

где

$$
\Psi_{r}(s)=\sqrt{\frac{\left(A_{r}\right)_{12}}{\sin \mu_{r}}}\left(x_{r} \tilde{c}_{2}+y_{r} \tilde{s}_{2}\right),
$$

$$
x_{r}=c_{1}+\beta_{r} s_{1} ; \quad x y_{r}=\tau_{21} c_{1}-s_{1}+\beta_{r}\left(\tau_{21} s_{1}+c_{1}\right) .
$$

Из (18) найдем квадрат модуля функции Флоке:

в секторе 1

$$
\Phi_{r}(s)=\frac{\left(A_{r}\right)_{12}}{\sin \mu_{r}}\left[\tilde{c}_{1}^{2}+\left(\beta_{r}+\beta_{r}^{*}\right) \tilde{c}_{1} \tilde{s}_{1}+\beta_{r} \beta_{r}^{*} \tilde{s}_{1}^{2}\right]
$$

в секторе 2

$$
\Phi_{r}(s)=\frac{\left(A_{r}\right)_{12}}{\sin \mu_{r}}\left[x_{r} x_{r}^{*} \tilde{c}_{2}^{2}+\left(x_{r} y_{r}^{*}+x_{r}^{*} y_{r}\right) \tilde{c}_{2} \tilde{s}_{2}+y_{r} y_{r}^{*} \tilde{s}_{2}^{2}\right] \text {. }
$$


54

Ю. Лембра

При проведении численных расчетов удобно пользоваться следующими формулами, вытекающими из (15), (16)- и (19):

$$
\begin{aligned}
& \beta_{r}+\beta_{r}^{*}=\frac{2 r\left[\cos \mu_{r}-\left(A_{r}\right)_{11}\right]}{\left(A_{r}\right)_{12}}=\frac{r\left[\left(A_{r}\right)_{22}-\left(A_{r}\right)_{11}\right]}{\left(A_{r}\right)_{12}} \\
& \beta_{r} \beta_{r}^{*}=\frac{r^{2}}{\left(A_{r}\right)_{12}^{2}}\left[1-2\left(A_{r}\right)_{11} \cos \mu_{r}+\left(A_{r}\right)_{11}^{2}\right]=-\frac{r^{2}\left(A_{r}\right)_{21}}{\left(A_{r}\right)_{12}} \\
& x_{r} x_{r}^{*}=c_{1}^{2}+s_{1} c_{1}\left(\beta_{r}+\beta_{r}^{*}\right)+s_{1}^{2} \beta_{r} \beta_{r}^{*} \\
& \left.\begin{array}{l}
x\left(x_{r} y_{r}^{*}+x_{r}^{*} y_{r}\right)=2 c_{1}\left(\tau_{21} c_{1}-s_{1}\right)+\left(2 \tau_{21} s_{1} c_{1}+c_{1}^{2}-s_{1}^{2}\right)\left(\beta_{r}+\beta_{r}^{*}\right)+ \\
\left.x^{2} y_{r} y_{r}^{*}=\left(\tau_{21} c_{1}-s_{1}\right)^{2}+\left(\tau_{21} c_{1}-s_{1}\right)\left(\tau_{21} s_{1}+c_{1}\right) \beta_{r} \beta_{r}^{*}+c_{1}\right)\left(\beta_{r}+\beta_{r}^{*}\right)+\left(\tau_{21} s_{1}+c_{1}\right)^{2} \beta_{r} \beta_{r}^{*} .
\end{array}\right\}
\end{aligned}
$$

Целесообразно представить (20) в виде:

В секторе 1

$$
\Phi_{r 1}=\frac{\left(A_{r}\right)_{12}}{\sin \mu_{r}}\left\{1+\frac{1}{2} \sqrt{\left(\beta_{r}+\beta_{r}^{*}\right)^{2}+\left(\beta_{r} \beta_{r}^{*}-1\right)^{2}}\left[\cos \varphi_{1}-\cos \left(2 v+\varphi_{1}\right)\right]\right\}\left(v=\frac{s}{r}\right),
$$

в секторе 2

$$
\left.\begin{array}{c}
\Phi_{r 2}=\frac{\left(A_{r}\right)_{12}}{\sin \mu_{r}}\left\{x_{r} x_{r}^{*}+\frac{1}{2} \sqrt{\left(x_{r} y_{r}^{*}+x_{r}^{*} y_{r}\right)^{2}+\left(y_{r} y_{r}^{*}-x_{r} x_{r}^{*}\right)^{2}\left[\cos \varphi_{2}-\right.}\right. \\
\left.\left.-\cos \left(2 v+\varphi_{2}\right)\right]\right\}\left(v=\frac{\gamma s}{r}\right)
\end{array}\right\}
$$

где

$$
\operatorname{tg} \varphi_{1}=\frac{\beta_{r}+\beta_{r}^{*}}{\beta_{r} \beta_{r}^{*}-1}, \operatorname{tg} \varphi_{2}=\frac{x_{r} y_{r}^{*}+x_{r}^{*} y_{r}}{y_{r} y_{r}^{*}-x_{r} x_{r}^{*}}
$$

Для сравнения представляет интерес рассмотреть гипотетический случай $\operatorname{tg} \gamma_{12}=\operatorname{tg} \gamma_{21}=0$. В этом случае выведенные формулы упрощаются. Подставляя $\tau_{12}=\tau_{21}=0$ в (15)-(17), (21) и (23), найдем:

$$
\begin{aligned}
& \cos \mu_{r}=c_{1} c_{2}-\frac{\left(1+x^{2}\right)}{2 \varkappa} s_{1} s_{2}, \quad C_{r}=\sqrt{\frac{r\left(x s_{1} c_{2}+c_{1} s_{2}\right)}{\varkappa \sin \mu_{r}}} \\
& \beta_{r}+\beta_{r}^{*}=-\varkappa\left(x_{r} y_{r}^{*}+x_{r}^{*} y_{r}\right)=\frac{\left(1-\chi^{2}\right) s_{1} s_{2}}{\chi s_{1} c_{2}+c_{1} s_{2}} \\
& \beta_{r} \beta_{r}^{*}-1=-\frac{\left(1-x^{2}\right) c_{1} s_{2}}{x s_{1} c_{2}+c_{1} s_{2}}, \quad x\left(y_{r} y_{r}^{*}-x_{r} x_{r}^{*}\right)=\frac{\left(1-x^{2}\right) s_{1} c_{2}}{\varkappa s_{1} c_{2}+c_{1} s_{2}} \\
& x_{r} x_{r}^{*}=1, \quad \varphi_{1}=\pi-v_{1}, \quad \varphi_{2}=2 \pi-v_{2} .
\end{aligned}
$$


Подставляя (24) в (22), получим:

$$
\left.\begin{array}{l}
\Phi_{r 1}=\frac{r}{\sin \mu_{r}}\left\{s_{1} c_{2}+\frac{c_{1} s_{2}}{x}+\frac{\left(1-x^{2}\right) s_{2}}{2 \varkappa}\left[\cos \left(2 v-v_{1}\right)-c_{1}\right]\right\}\left(v=\frac{s}{r}\right) \\
\Phi_{r 2}=\frac{r}{\sin \mu_{r}}\left\{s_{1} c_{2}+\frac{c_{1} s_{2}}{x}-\frac{\left(1-x^{2}\right) s_{1}}{2 x^{2}}\left[\cos \left(2 v-v_{2}\right)-c_{2}\right]\right\}\left(v=\frac{x s}{r}\right) .
\end{array}\right\}
$$

Из (25) видно, что огибающая имеет максимум в середине сектора 1 и мннимум в середине сектора 2. Полученный результат аналогичен хорошо известному из теорни сильнофокусирующих ускорителей обстоятельству, что частица находится в фокусирующих секторах дальше от равновесной орбиты, чем в дефокусирующих секторах.

Из теорни обычных слабофокусирующих ускорителей известно, что фокусирующие силы изменяются в случае однородного магнитного поля пропорционально напряженности магнитного поля и обратно пропорционально радиусу равновесной орбиты (см., напр., []). Применяя это правило в нашем случае, убедимся, что в секторе 2 фокусирующие силы в $\frac{1}{x^{2}}$ раз меньше, чем в секторе 1. Теперь становится понятным

смысл вытекаюшего из (25) факта, что частица находится в секторе 1 дальше от равновесной орбиты, чем в секторе 2. Действительно, в секторе 2 на частицу действуют менее сильные фокусирующие силы и частица попадает в сектор 1 наиболее отклонепной. С другой стороны, на находящуюся в секторе 1 частицу действуют более сильные фокусирующие силы и она попадает в сектор 2 на меньшем расстоянин от равновесной орбиты, чем в секторе 1.

В случае $\operatorname{tg} \gamma_{12} \neq 0$ и $\operatorname{tg} \gamma_{21} \neq 0$ возникают фокусирующие и дефокусирующие снлы, обусловленные краевым магнитным полем, и экстремумы огибающей смещаются.

\section{3. Вертикальные колебания}

В первом приближенни можно считать для уравнения (1)

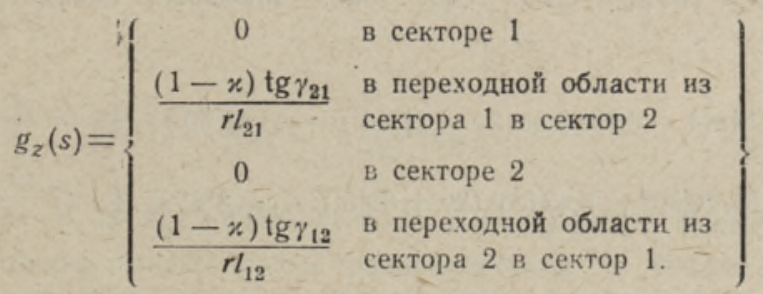

Расчеты вполне аналогичны проведенным в п. 2 расчетам. Поэтому мы их повторять не будем и приводим сразу окончательный результат:

в секторе 1

$$
\Phi_{z 1}=\frac{\left(A_{z}\right)_{12}}{\sin \mu_{z}}\left[1+\left(\beta_{z}+\beta_{z}^{*}\right) v+\beta_{z} \beta_{z}^{*} v^{2}\right] \quad\left(v=\frac{s}{r}\right)
$$

в секторе 2

$$
\Phi_{z 2}=\frac{\left(A_{z}\right)_{12}}{\sin \mu_{z}}\left[x_{z} x_{z}^{*}+\left(x_{z} y_{z}^{*}+x_{z}^{*} y_{z}\right) v+y_{z} y_{z}^{*} v^{2}\right] \quad\left(v=\frac{x s}{r}\right),
$$


где

$$
\begin{aligned}
& \left(A_{z}\right)_{11}=1-\frac{\tau_{21} v_{3}}{x} ; \quad \frac{\left(A_{z}\right)_{12}}{r}=v_{1}-\frac{\tau_{21} v_{1} v_{2}}{x}+\frac{v_{2}}{x} \\
& r\left(A_{z}\right)_{21}=-\left(\tau_{12}+\tau_{21}\right)+\frac{\tau_{12} \tau_{21} \nu_{2}}{x} \\
& \left(A_{z}\right)_{22}=1-\tau_{12}\left(v_{1}+\frac{v_{2}}{x}\right)-\tau_{21} v_{1}+\frac{\tau_{12} \tau_{21}}{\psi} v_{1} v_{2} \\
& \cos \mu_{z}=\frac{\left(A_{z}\right)_{11}+\left(A_{z}\right)_{22}}{2}=1-\frac{\tau_{12}+\tau_{21}}{2}\left(\nu_{1}+\frac{\nu_{2}}{x}\right)+\frac{\tau_{12} \tau_{21} v_{1} v_{2}}{2 x} \\
& \begin{aligned}
\beta_{z}+\beta_{z}^{*} & =\frac{2 r\left[\cos \mu_{z}-\left(A_{z}\right)_{11}\right]}{\left(A_{z}\right)_{12}}=\frac{r\left[\left(A_{z}\right)_{22}-\left(A_{z}\right)_{11}\right]}{\left(A_{z}\right)_{12}} \\
\beta_{z} \beta_{z}^{*} & =\frac{r^{2}}{\left(A_{z}\right)_{12}^{2}}\left[1-2\left(A_{z}\right)_{11} \cos \mu_{z}+\left(A_{z}\right)_{11}^{2}\right]=-\frac{r^{2}\left(A_{z}\right)_{21}}{\left(A_{z}\right)_{13}}
\end{aligned} \\
& x_{z} x_{z}^{*}=1+\left(\beta_{z}+\beta_{z}^{*}\right) v_{1}+\beta_{z} \beta_{z}^{*} v_{1}^{2} \\
& x\left(x_{z} y_{z}^{*}+x_{z}^{*} y_{z}\right)=-2 \tau_{21}+\left(1-2 v_{1} \tau_{21}\right)\left(\beta_{z}+\beta_{z}^{*}\right)+2 v_{1}\left(1-v_{1} \tau_{21}\right) \beta_{z} \beta_{z}^{*} \\
& x^{2} y_{z} y_{z}^{*}=\tau_{21}^{2}-\tau_{21}\left(1-v_{1} \tau_{21}\right)\left(\beta_{z}+\beta_{z}^{*}\right)+\left(1-v_{1} \tau_{21}\right)^{2} \beta_{z} \beta_{z}^{*} .
\end{aligned}
$$

Как видно из-(27), экстремумы огибающей для вертикальных колебаний являются минимумами.

\section{4. Пример}

В качестве иллюстрации приведем пример со следующими значениями параметров: $x=0,3, v_{1}=1,417 \mathrm{rad}, v_{2}=0,1544 \mathrm{rad}, \operatorname{tg} \gamma_{12}=-0,877, \operatorname{tg} \gamma_{21}=1,25$. Эти параметры получены нами при расчете одного из вариантов секторного циклотрона с числом элементов периодичности на оборот $N=4$. Tогда по (16) и (28) $\cos \mu_{r}=-0,123$ и $\cos \mu_{z}=0,550$. При таких значениях параметров (22) н (27) принимают вид:

$$
\left.\begin{array}{cc}
\Phi_{r 1}=1,51 r\left\{1+0,295\left[\cos \left(2 v-21^{\circ} 30^{\prime}\right)-\cos 21^{\circ} 30^{\prime}\right]\right\} & \left(v=\frac{s}{r}\right) \\
\Phi_{r 2}=1,51 r\left\{0,497-5,42\left[\cos \left(2 v+8^{\circ} 45^{\prime}\right)-\cos 8^{\circ} 45\right]\right\} & \left(v=\frac{x s}{r}\right) \cdot v
\end{array}\right\}
$$

График функций $\left(22^{\prime}\right)$ и $\left(27^{\prime}\right)$ представлен на фиг. 1.

Анализ $\left(22^{\prime}\right)$, $\left(27^{\prime}\right)$ и фиг. 1 указывает на следующую закономерность: функция $\Phi_{r, z}$ принимает максимальные значения вблизи граничной линин, где имеется фокусировка краевым магнитным полем, а минимальное значение вблизи граничной линии, где имеется дефокусировка краевым магнитным полем. Это физически понятно, учитывая рассуждения, приведенные в конце п. 2. Из физических соображенин̆ ясно, что аналогичное поведение функции $\Phi_{r, z}$ встречается и при других значениях параметров. 
По $(6),\left(22^{\prime}\right)$ и $\left(27^{\prime}\right)$ найдем эффективные радиусы секторного циклотрона в нашем прнмере:

$$
\begin{aligned}
& \left(R_{r}\right)_{\text {эфф }}=1,54 r \\
& \left(R_{r}\right)_{9 \phi \Phi}=2,85 r .
\end{aligned}
$$

Как указано в ['], для сравнения ускорнтелей разных типов физически разумно сравнить отношение $R_{\text {эфф }}$ к линейным размерам зазора магнита. Так, например, можно сравннвать отношения $R_{\text {эфф }} / R_{\text {м }}$, где $R_{\text {м }}-$ максимальное расстояние от равновесной орбиты до центра ускорителя. Используя данное в $[3,4]$ выражение для $R_{\mathrm{M}}$ найдем $\left(R_{r}\right)_{\text {эфф }} / R_{\mathrm{M}}=1,23$ и $\left(R_{z}\right)_{\text {эфф }} / R_{\mathrm{M}}=2,28$. Напомним, что в слабо. фокусирующих ускорителях $R_{\text {эфф }} / R_{\mathrm{M}}>1$, а в сильнофокусирующих ускорителях порядок величины $R_{\text {эфф }} / R \simeq 0,1$. Такое сопоставление показывает, что по сравнению с сильнофокуснрующими ускорителями секторный циклотрон обладает худшими фокусирующими свойствами. Это понятно, так как в секторном циклотроне данного варианта используется однородное магнитное поле и только для получення вертнкальной фокусировки введены краевые магнитные поля. Поэтому параметры, характеризующие его фокусирующие свойства, одного порядка с соответствующими параметрами в обычных слабофокусирующих ускорителях.

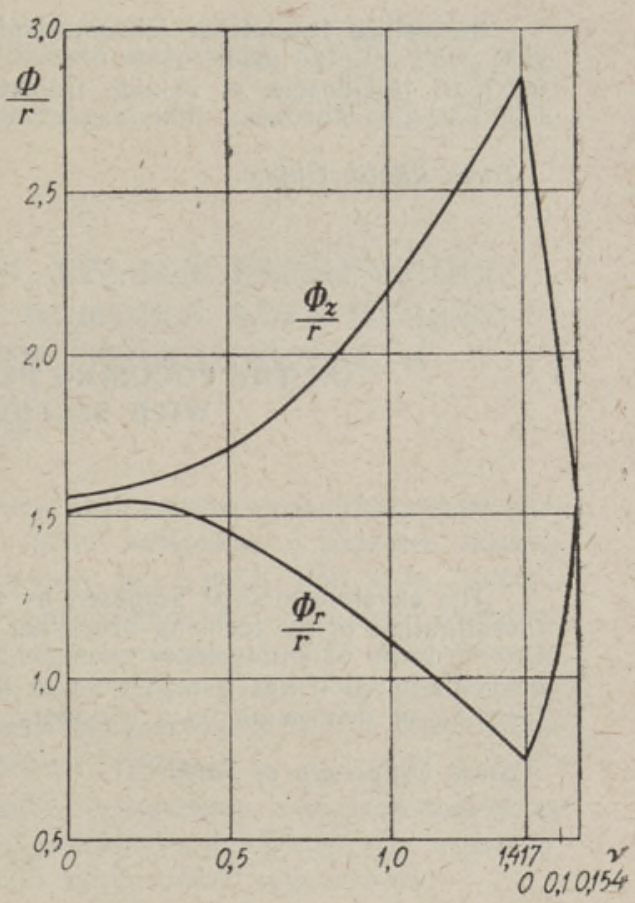

Фнr. 1.

Автор выражает благодарность Д. М. Каминкеру за обсуждение результатов зтой работы.

\section{ЛИ ТЕ Р А Т У Р А}

1. А. М. Балдин, В. В. Мих айлов, М. С. Р абинович, ЖЭТФ, 31, 993, 1956.

2. М. С. Р а би но в и ч, Основы теории сннхрофазотрона. Тр. ФИАН, т. 10, М., 1958.

3. Е. М. Моро з, М. С. Р а би но в и ч, Proc. CERN Symp., 1, 547, 1956; см. также приложение № 4 к журналу «Атомная энергия», 27, 1957.

4. Е. M. Мороз, М. С. Р а 6 инов и ч, Приборы и техника эксперимента. № 1, $15,1957$.

5. Дж. Стокер, Нелинейные колебания в механических и электрических системах. ИЛ, 1953.

6. J. T e i c h m a n, Czechosl. Journ. Phys., 9, 47, 1959. 


\section{SEKTORILISE TSUKLOTRONI FOKUSSEERIVATEST OMADUSTEST}

\section{J. Lembra}

\section{Resümee}

Sektorilise tsüklotroni fokusseerivaid omadusi uuriti Baldini, Mihhailovi ja Rabinovitši poolt esitatud mähisjoonte meetodil. Numbrilise näite ja füüsikaliste kaalutluste põhjal jôuti järeldusele, et ioonide trajektoori mähisjoone maksimum (miinimum) paikneb selle äärjoone läheduses, mille magnetväli mõjub ioonidele fokusseerivalt (defokusseerivalt).

Tartu Riiklik Olikool

Saabus toimetusse

26. VIII 1959

\section{ON THE FOCUSING PROPERTIES OF THE CYCLOTRON WITH SECTIONED MAGNET SYSTEM}

\section{J. Lembra}

\section{Summary}

The envelope-method proposed by Baldin, Mikhailov and Rabinovich is used for the investigation of the focusing properties of the cyclotron with a sectioned magnet system. On the basis of a numerical example and physical conceptions, it is concluded that the envelope-function has a maximum or minimum close to the boundary line where the focusing or defocusing by a boundary magnetic field exists. 